PROCEEDINGS OF THE

AMERICAN MATHEMATICAL SOCIETY

Volume 133, Number 11, Pages 3361-3370

S 0002-9939(05)07913-X

Article electronically published on May 9, 2005

\title{
CAUCHY TRANSFORMS OF CHARACTERISTIC FUNCTIONS AND ALGEBRAS GENERATED BY INNER FUNCTIONS
}

\author{
ALEC L. MATHESON AND MICHAEL I. STESSIN
}

(Communicated by Joseph A. Ball)

\begin{abstract}
We prove that Cauchy transforms of characteristic functions of subsets of positive measure of the unit circle are equidistributed in the unit disk in the sense that the $L^{p}$-closure of the polynomial algebra in these Cauchy transforms coincides with the $L^{p}$-closure of the polynomial algebra in a canonical inner function. As a corollary to this result we find conditions describing when the polynomial algebra in two singular inner functions determined by point masses is dense in the Hardy spaces $H^{p}$.
\end{abstract}

\section{INTRODUCTION}

Let $\mathcal{A}$ be a subalgebra of $H^{\infty}$. It can be shown that the structure of the lattice of closed subspaces of $H^{p}, p<\infty$, invariant under the multiplication by elements of $\mathcal{A}$, is closely related to the structure of the $H^{p}$-closure of $\mathcal{A}$ (cf. [7]). This leads to Beurling-type results for multiplication invariant subspaces. In particular, when $\mathcal{A}$ is dense in $H^{p}$ the lattice of $\mathcal{A}$-invariant subspaces coincides, at least in a weak sense, with the lattice of $z$-invariant subspaces, and is described by the theorem of Beurling [2].

Results about the density in the disk-algebra when $\mathcal{A}$ is a subalgebra of the disk algebra were obtained by J. Wermer, N. Sibony and J. Wermer, R. Blumenthal and others (cf. [3], [11], [13], [14], [8]). An obvious necessary condition for density is the separation of points of the disk. Simple examples show that this condition by itself is not enough: boundary behavior has to be considered, too. It was assumed in the papers mentioned above that the generators of the algebra are analytic in the closed domain, and then the condition additional to point separation was in some form of non-vanishing of the derivatives on the boundary.

The case of finitely generated polynomial algebras in the Hardy spaces was considered in [7]. Under certain conditions on generators the polynomial algebra has finite codimension in $H^{p}$. The result in [7] gives an estimate of this codimension.

If $\mathcal{A}$ is finitely generated but some of the generators are not disk algebra functions, the methods of the previously mentioned papers are not applicable. The case of an algebra generated by two functions, one of which is a finite Blaschke product, and the other is an arbitrary bounded analytic function in the unit disk,

Received by the editors May 5, 2004 and, in revised form, June 28, 2004.

2000 Mathematics Subject Classification. Primary 46J10; Secondary 46J15, 30D50, 30D55.

This work was accomplished while the first author was visiting the University at Albany. He thanks that institution for the hospitality extended during his visit.

(C)2005 American Mathematical Society Reverts to public domain 28 years from publication 
was considered in [12]. The condition for density in $H^{p}$ was expressed in terms of a certain function $\Gamma$ associated with these generators.

In this paper we consider a situation when both generators are discontinuous at some points of the boundary. Namely, let $w_{1}$ and $w_{2}$ be two points in $\mathbb{T}$, and let $g_{1}$ and $g_{2}$ be singular inner functions determined by point masses $\lambda_{1}$ and $\lambda_{2}$ at $w_{1}$ and $w_{2}$, respectively. Is $\mathbb{C}\left[g_{1}, g_{2}\right]$ dense in $H^{p}$ ? None of the previously-developed methods works in this situation: irregular boundary behavior does not allow the application of Cartan's theorems as was done in 7] (this is impossible even when one of the generators is discontinuous on the boundary). Moreover, both $g_{1}$ and $g_{2}$ have infinite multiplicity in $\mathbb{D}$ which makes it impossible to define $\Gamma$ as in $[12$.

Our first result is peripheral to the main result of this paper, but is of independent interest.

Theorem 1. Let $f$ be a function of bounded mean oscillation on $\mathbb{T}$, and let $\Xi$ be the smallest $\sigma$-algebra with respect to which $f$ is measurable. Then the algebra $\mathbb{C}[f, \bar{f}]$ is dense in the closed subspace of $L^{p}$ consisting of functions measurable with respect to $\Xi$.

This theorem is a simple consequence of a classical moment argument and the John-Nirenberg Theorem. We will also show that it is sharp in the sense that there exist functions in $L_{+}^{\infty}=\bigcap_{p<\infty} L^{p}$ for which the conclusion fails.

Let $A$ be a subset of the unit circle $\mathbb{T}$ such that $0<|A|<1$. the Cauchy transform

$$
C \chi_{A}(z)=\int_{A} \frac{d m(\zeta)}{1-\bar{\zeta} z}
$$

has real part

$$
\frac{|A|}{2}+\frac{1}{2} \int_{A} \frac{1-|z|^{2}}{|\zeta-z|^{2}} d m(\zeta) .
$$

This harmonic function has boundary values $(1+|A|) / 2$ a.e. on $A$ and $|A| / 2$ a.e. on the complement of $A$. Hence $C \chi_{A}$ maps the unit disk into the strip $|A| / 2<$ $\Re z<(1+|A|) / 2$. Let $\psi$ be the Riemann mapping of this strip onto the unit circle, taking the vertical line $\{\Re z=(1+|A|) / 2\}$ onto the upper semicircle, and which is real for real $z$. Then $g=\psi \circ C \chi_{A}$ is inner. Our next theorem states that $g$ can be approximated in every $H^{p}, 1 \leq p<\infty$, by polynomials in $C \chi_{A}$. Of course the approximation of $C \chi_{A}$ by polynomials in $g$ follows from the boundedness of the operator of composition with $g$ acting on $H^{p}$.

Theorem 2. Let $A \in \mathbb{T}$, with $0<|A|<1$ (where, as usual, $|A|$ stands for the normalized Lebesgue measure of $A$ ). With $g=\psi \circ C \chi_{A}$ as above, $g$ is an inner function such that $g^{-1}(\mathbb{T} \cap\{\Im z>0\})=A(\bmod 0)$ and $g^{-1}(\mathbb{T} \cap\{\Im z<0\})=A^{c}$ $(\bmod 0)$. Then

(a) $\overline{\mathbb{C}[g]}=\overline{\mathbb{C}\left[C \chi_{A}\right]}$.

(b) $g$ is analytically extendable across $\mathbb{T} \backslash \partial A$; consequently, spec $g \subset \partial A$.

(c) $g$ is a Blaschke product of order $n$ if and only if $A$ is a union of $n$ separated arcs.

Remark. Theorem 2 allows us to answer positively the following question on cluster sets posed by Alexander Izzo [6]. Given a Borel set $A$ with $0<|A|<1$, does there exist a bounded function such that $f$ is continuous on $A^{c}$, and for each $\zeta \in \mathbb{T}$, the cluster set of $f$ at $\zeta$ is closed under complex conjugation? 
Theorem 2 reduces the problem of density of the algebra generated by two pointmass singular functions to a certain pure geometric consideration. As a result we prove the following theorem.

Theorem 3. Let $w_{1}, w_{2} \in \mathbb{T}, \lambda_{1}, \lambda_{2}>0, g_{i}=\exp \left\{\lambda_{i} \frac{z+w_{i}}{z-w_{i}}\right\}, i=1,2$. If

$$
\frac{\left(1-|a|^{2}\right)^{2}}{\left|1-\bar{a} w_{1}\right|^{2}\left|1-\bar{a} w_{2}\right|^{2}} \lambda_{1} \lambda_{2}<\pi^{2},
$$

where $a$ is the midpoint of the geodesic joining $w_{1}$ and $w_{2}$, then the algebra $\mathbb{C}\left[g_{1}, g_{2}\right]$ is dense in $H^{p}, 1 \leq p<\infty$. If the above expression is greater than $\pi^{2}$, then $\mathbb{C}\left[g_{1}, g_{2}\right]$ is not dense in $H^{p}$.

The structure of this paper is as follows. In section 2 we prove Theorem 1 . In section 3 we prove Theorem 2 and its corollary and provide our answer to Izzo's question. Section 4 is devoted to proof of Theorem 3 . Finally, section 5 contains several open problems.

\section{Subalgebras associated with $B M O$}

In order to prove Theorem 1 we make use of the following proposition. This is a simple two-variable extension of standard results on orthogonal polynomials. The elementary proof given below is adapted from [5].

A function on the unit circle $\mathbb{T}$ is said to have bounded mean oscillation if

$$
\|f\|_{*}=\sup _{I} \frac{1}{|I|} \int_{I}\left|f-f_{I}\right| d m<\infty,
$$

where the supremum is taken over all subarcs $I$ of $\mathbb{T}$, and $f_{I}$ is the mean of $f$ over $I$, that is,

$$
f_{I}=\frac{1}{|I|} \int_{I} f d m .
$$

Here $|I|=m(I)$. We let $B M O$ denote the class of functions of bounded mean oscillation. Clearly bounded functions are contained in $B M O$. It is easy to see that the unbounded function $|\log | \theta||$ is also in $B M O$. The classic estimate of John and Nirenberg shows that log is typical of unbounded functions in $B M O$.

Theorem $\mathbf{C}$ (John-Nirenberg). If $f \in B M O$, then for every arc $I \subset \mathbb{T}$,

$$
m\left(\left\{\theta \in I:\left|f(\theta)-f_{I}\right|>\lambda\right\}\right) \leq C e^{-c \frac{\lambda}{\pi f \|_{*}}} \cdot|I|
$$

for all $\lambda>0$, where $C$ and $c$ are absolute constants.

It is an immediate consequence that $B M O$ is contained in $L^{p}$ for every $p<\infty$. We let $B M O A$ be the class of functions in $B M O$ which have analytic extensions to $\mathbb{D}$. Hence $B M O A \subset H^{p}$ for each $p<\infty$. A proof of Theorem $\mathrm{C}$ and other basic propereties of $B M O$ can be found in [4].

Proposition 1. Let $\mu$ be a positive measure on the plane which satisfies the growth estimate $\mu(\{|z|>r\})<C e^{-\lambda r}$ for all $r>0$, where $\lambda$ is a positive constant. Let $f \in L^{p}(\mu)$ for some $1<p \leq \infty$. Suppose $f$ satisfies

$$
\int x^{m} y^{n} f(x, y) d \mu(x, y)=0
$$

for all nonnegative integers $m$ and $n$. Then $f=0$ almost everywhere with respect to $\mu$. 
Proof. An application of Hölder's inequality shows that the function

$$
F(z, w)=\int e^{i x z} e^{i y w} f(x, y) d \mu(x, y)
$$

is holomorphic for $|\Im z|<\lambda,|\Im w|<\lambda$. The moment condition implies that all the complex derivatives of $F$ vanish at the origin, and hence $F$ is identically zero. Thus

$$
\int e^{i x s} e^{i y t} f(x, y) d \mu(x, y)=F(s, t)=0
$$

for all real numbers $s$ and $t$. By uniqueness of the Fourier transform, it follows that $f=0$ almost everywhere with respect to $\mu$.

Proof of Theorem 1. Now suppose $f \in B M O$, with associated $\sigma$-algebra $\Xi$. We denote by $\mu$ the pull-back measure of $f$, that is, for each open $G \subset \mathbb{C}, \mu(G)=$ $m(\{z \in \mathbb{T}: f(z) \in G\})$.

Now $\phi \in L^{1}(\mathbb{T})$ is measurable with respect to $\Xi$ if and only if $\phi=h(f, \bar{f})$ for some $h \in L^{1}(\mu)$. Moreover

$$
\int_{\mathbb{T}}|\phi|^{p} d m=\int_{\mathbb{C}}|h|^{p} d \mu
$$

for every $1 \leq p<\infty$. Thus $\mathbb{C}[f, \bar{f}]$ is dense in the closed subspace of $L^{p}$ consisting of $\Xi$-measurable functions if and only if polynomials in $z$ and $\bar{z}$ are dense in $L^{p}(\mu)$. If $\mathbb{C}[z, \bar{z}]=\mathbb{C}[x, y]$ is not dense in $L^{p}(\mu)$, there is, by the Hahn-Banach Theorem, a nonzero $h$ in $L^{q}(\mu), 1 / p+1 / q=1$, such that

$$
\int x^{m} y^{n} h(x, y) d \mu(x, y)=0
$$

for all nonnegative integers $m$ and $n$. By the John-Nirenberg theorem, the measure $\mu$ decays exponentially, so the existence of such an $h$ contradicts Proposition 1

The following example shows that the condition $f \in B M O$ is sharp. Let

$$
\varphi(x)=\lambda \int_{0}^{x} e^{-\sqrt[4]{t}} d t
$$

and $f(x)=\varphi^{-1}(x)$, where

$$
\lambda=\frac{2 \pi}{\int_{0}^{\infty} e^{-\sqrt[4]{t}} d t} .
$$

Then $f$ is defined and real-valued on $[0,2 \pi)$ and $f^{\prime}=\frac{1}{\lambda} e^{\sqrt[4]{f}}$. It is very easy to check that $f \in L^{p}[0,2 \pi]$ for every $0<p<\infty$. Now, for every $n$ we have (see [9, p. 22] for the last equality)

$$
\int_{0}^{2 \pi} \sin \sqrt[4]{f(x)} f(x)^{n} d x=\lambda \int_{0}^{\infty} t^{n} e^{-\sqrt[4]{t}} \sin \sqrt[4]{t} d t=0 .
$$

This shows that the function $\sin \sqrt[4]{f}$ is orthogonal to every polynomial in $f$ and $\bar{f}$. This function is clearly in every $L^{p}$ and measurable with respect to the $\sigma$-algebra generated by $f$. This shows that the condition $f \in B M O$ cannot be replaced by the condition $f \in L_{+}^{\infty}$. 


\section{CAUChy transforms of CharaCteristic FUnCtions}

All functions discussed in this section are considered to be defined on, or restricted to, the unit circle $\mathbb{T}$.

Let $A \subset \mathbb{T}, m(A)>0$, and let $\hat{f}$ be the Cauchy transform of $\chi_{A}$, that is,

$$
\hat{f}(z)=\int_{A} \frac{d m(w)}{1-z \bar{w}}, \quad z \in \mathbb{D} .
$$

It is well known that $\hat{f}$ is in $B M O A$, and therefore can be identified with its boundary values. Write $f=\hat{f}-m(A)$, then we obviously have

$$
\chi_{A}=\lambda+f+\bar{f}
$$

where $\lambda=m(A)$ is a real constant.

Proposition 2. For every $k, l \geq 0$

$$
f^{k} \bar{f}^{l}=\frac{(-1)^{l} k}{k+l} f^{k+l}+\frac{(-1)^{k} l}{k+l} \bar{f}^{k+l}+r_{k, l} \circ f+q_{k, l} \circ \bar{f},
$$

where $r_{k, l}$ and $q_{k, l}$ are polynomials of degree $k+l-1$, and $q_{k, l}(0)=0$. Consequently, the Cauchy transform of a polynomial in $f$ and $\bar{f}$ is a polynomial in $f$.

Proof. Obviously $\chi_{A}^{2}=\chi_{A}$. By (1) this can be written as

$$
\lambda^{2}+f^{2}+\bar{f}^{2}+2 \lambda f+2 \lambda \bar{f}+2 f \bar{f}=\lambda+f+\bar{f} .
$$

Solving the last equation for $f \bar{f}$ we see that

$$
f \bar{f}=-\frac{1}{2} f^{2}-\frac{1}{2} \bar{f}^{2}+r_{1,1} \circ f+q_{1,1} \circ \bar{f},
$$

where $r_{1,1}(z)=\left(\lambda-\lambda^{2}\right)+(1-2 \lambda) z$ and $q_{1,1}(z)=(1-2 \lambda) z$. The relation $(3)$ establishes $(2)$ for the case $k+l=2$. We continue by induction in $k+l$. Suppose that (2) holds for all $k, l$ such that $k+l \leq m-1$. We have

$$
\chi_{A}^{m}=\chi_{A}
$$

and

This implies

$$
(\lambda+f+\bar{f})^{m}=\lambda+f+\bar{f} .
$$

$$
(f+\bar{f})^{m}=\mathcal{S}_{m-1}(f, \bar{f}),
$$

where $\mathcal{S}_{m-1}(f, \bar{f})$ is a polynomial of total degree $m-1$ in $f$ and $\bar{f}$. By the induction assumption

$$
\mathcal{S}_{m-1}(f, \bar{f})=t_{m-1}(f)+u_{m-1}(\bar{f})
$$

where $t_{m-1}$ and $u_{m-1}$ are polynomials of degree $m-1$ and $u_{m-1}(0)=0$. Thus,

$$
f^{m}+\bar{f}^{m}+\sum_{k=1}^{m-1}\left(\begin{array}{c}
m \\
k
\end{array}\right) f^{k} \bar{f}^{m-k}=t_{m-1}(f)+u_{m-1}(\bar{f}) .
$$

Using the induction assumption once again we obtain

$$
\begin{aligned}
m f^{m-1} \bar{f}= & -f^{m}-\bar{f}^{m}-\bar{f} \sum_{k=1}^{m-2}\left(\begin{array}{c}
m \\
k
\end{array}\right)\left[\left(\frac{(-1)^{m-k-1} k}{m-1} f^{m-1}\right)\right. \\
& \left.+\left(\frac{(-1)^{k}(m-k-1)}{m-1} \bar{f}^{m-1}\right)\right]+\tilde{\mathcal{S}}_{m-1}(f, \bar{f})+t_{m-1}(f)+u_{m-1}(\bar{f}),
\end{aligned}
$$


where $\tilde{\mathcal{S}}_{m-1}(f, \bar{f})$ is another polynomial in $f$ and $\bar{f}$ of total degree $m-1$. An argument similar to the one above yields

$$
\begin{aligned}
m f^{m-1} \bar{f}=-f^{m}-\bar{f}^{m}-\bar{f}^{m} \sum_{k=1}^{m-2}(-1)^{k}\left(\begin{array}{c}
m \\
k
\end{array}\right) \frac{m-k-1}{m-1} & \\
& -f^{m-1} \bar{f} \sum_{k=1}^{m-2}(-1)^{m-k-1}\left(\begin{array}{c}
m \\
k
\end{array}\right) \frac{k}{m-1}+\tilde{t}_{m-1}(f)+\tilde{u}_{m-1}(\bar{f}),
\end{aligned}
$$

where again $\tilde{t}_{m-1}$ and $\tilde{u}_{m-1}$ are polynomials of degree $m-1$ and $\tilde{u}_{m-1}(0)=0$. An easy computation shows that

$$
\begin{aligned}
& \sum_{k=1}^{m-2}\left(\begin{array}{c}
m \\
k
\end{array}\right)(-1)^{k} \frac{m-k-1}{m-1}=-1+\frac{(-1)^{m}}{m-1}, \\
& \sum_{k=1}^{m-2}\left(\begin{array}{c}
m \\
k
\end{array}\right)(-1)^{m-k-1} \frac{k}{m-1}=-m+\frac{m}{m-1} .
\end{aligned}
$$

Now, (4) and (5) yield

$$
\frac{m}{m-1} f^{m} \bar{f}=-f^{m}+\frac{(-1)^{m-1}}{m-1} \bar{f}^{m}+\tilde{t}_{m-1} \circ f+\tilde{u}_{m-1} \circ \bar{f},
$$

and, finally,

$$
f^{m-1} \bar{f}=\frac{(-1)(m-1)}{m} f^{m}+\frac{(-1)^{m-1}}{m} \bar{f}^{m}+r_{m-1,1} \circ f+q_{m-1,1} \circ \bar{f},
$$

where $r_{m-1,1}=\frac{(m-1) \tilde{t}_{m-1}}{m}$ and $q_{m-1,1}=\frac{(m-1) \tilde{u}_{m-1}}{m}$. The last relation is exactly the relation (2) for $k=m-1, l=1$.

Suppose that we have already proved (2) for all pairs $(k, l)$ such that $k+l=m$ and $1 \leq l \leq s$. Then we have by (3) that

$$
\begin{aligned}
f^{m-s} \bar{f}^{s} & =f^{m-s-1} \bar{f}^{s-1}|f|^{2} \\
& =f^{m-s-1} \bar{f}^{s-1}\left[-\frac{f^{2}}{2}-\frac{\bar{f}^{2}}{2}+r_{1,1} \circ f+q_{1,1} \circ \bar{f}\right] \\
& =\frac{(-1)^{s}(m-s)}{m} f^{m}+\frac{(-1)^{m-s} s}{m} \bar{f}^{m}+r_{m-s, s} \circ f+q_{m-s, s} \circ \bar{f} .
\end{aligned}
$$

By the induction assumption

$$
\begin{aligned}
& f^{m-s-1} \bar{f}^{s-1} r_{1,1} \circ f=v_{m-1} \circ f+w_{m-1} \circ \bar{f}, \\
& f^{m-s-1} \bar{f}^{s-1} q_{1,1} \circ \bar{f}=\tilde{v}_{m-1} \circ f+\tilde{w}_{m-1} \circ \bar{f},
\end{aligned}
$$

where $v_{m-1}, w_{m-1}, \tilde{v}_{m-1}, \tilde{w}_{m-1}$ are polynomials of degree $m-1$. Thus, since (2) was already proved for for $l \leq s,(6)$ implies

$$
\begin{aligned}
-\frac{1}{2} f^{m-s+1} \bar{f}^{s-1}-\frac{1}{2} f^{m-s-1} \bar{f}^{s+1}=\frac{(-1)^{s}(m-s)}{m} f^{m} & +\frac{(-1)^{m-s} s}{m} \bar{f}^{m} \\
& +\hat{r}_{m-1} \circ f+\hat{q}_{m-1} \circ \bar{f}
\end{aligned}
$$


and so

$$
\begin{aligned}
f^{m-s-1} \bar{f}^{s+1}=\frac{(-1)^{s+1}(m-s-1)}{m} f^{m}+ & \frac{(-1)^{m-s-1}(s+1)}{m} \bar{f}^{m} \\
& +r_{m-s-1, s+1} \circ f+q_{m-s-1, s+1} \circ \bar{f} .
\end{aligned}
$$

We are done.

Proof of Theorem 2. We note that $g=\psi \circ C \chi_{A}$ is a bounded function measurable with respect to the $\sigma$-algebra generated by $C \chi_{A}$. Since $f=C \chi_{A}$ is in $B M O A$, by Theorem $1, g$ is approximable by polynomials in $f$ and $\bar{f}$. By Proposition 2 , the Cauchy transform of a polynomial $P(f, \bar{f})$ has the form $C P(f, \bar{f})=Q(f)$, where $Q$ is a polynomial. Now (a) follows from the continuity of the Cauchy transform on $L^{p}(\mathbb{T})$.

Since $\mathbb{T} \backslash \partial A$ is open, it consists of the at most countable union of disjoint open arcs. The Cauchy transform of $\chi_{A}$ is analytically extendable across each such arc. The Schwarz reflection principle now implies (b).

Recall that the spectrum $\operatorname{spec}(g)$ of an inner function $g$ is the collection of points of the unit circle $\mathbb{T}$ which are limit points of zeros of $g$, or in the support of the measure which determines the singular part of $g$. Of course, $g$ is analytically extendable through some neighborhood of every point which is not in $\operatorname{spec}(g)$.

Now if $g$ is a Blaschke product of order $n$, then $A=g^{-1}(\mathbb{T} \cap \Im z>0)$ is a finite union of $n$ arcs. Conversely, if $A$ is a finite union of arcs, then the boundary spectrum of $g$ is empty. Indeed, by (b), $\mathbb{T} \backslash \partial A$ is not in the spectrum. Moreover, since the derivative of $g$ does not vanish on $\mathbb{T} \backslash \partial A, g$ is one-to-one on each component of $\mathbb{T} \backslash \partial A$ (cf. [1] ). This clearly implies that no point of $\partial A$ is in the boundary spectrum of $g$. Hence, $g$ is a finite Blaschke product with order equal to the number of components of $A$.

We can now answer Izzo's question from the Introduction. Given a Borel set $A$ with $0<|A|<1$, does there exist a bounded function such that $f$ is continuous on $A^{c}$, and for each $\zeta \in \mathbb{T}$, the cluster set of $f$ at $\zeta$ is closed under complex conjugation?

By regularity we may assume that $A$ is closed. By Theorem 2, the function $g=\psi \circ C \chi_{A}$ is inner, analytically extendable through every point of $\mathbb{T} \backslash A$ and satisfies the condition $g^{-1}(\mathbb{T} \cap\{\Re z \geq 0\})=A$. Let $\sigma$ be a singular probability measure whose support is the upper semicircle, which has a point mass at -1 and satisfies the condition

$$
\lim _{\theta \rightarrow 0^{-}} \int_{\mathbb{T}} \frac{1}{t-\theta} d \sigma\left(e^{i t}\right)<\infty .
$$

If $\phi$ is the inner function for which $\sigma$ is the Clark's measure at 1 , that is,

$$
\frac{1+\phi(z)}{1-\phi(z)}=\int \frac{\zeta+z}{\zeta-z} d \sigma(\zeta)
$$

then it is easy to check, using Aleksandrov's theorem [1], that $\phi$ is one-to-one on the lower semicircle, $\mathbb{T}_{-}$, is analytically extendible through every point of $\mathbb{T}_{-}$, and maps $\mathbb{T}_{-}$onto a proper subarc $I$ of $\mathbb{T}$. Let $h$ be a conformal map of the unit disk onto the disk slit over the radius from 0 to 1 and which takes $I$ onto this radius. Then the function $h \circ \phi \circ g$ satisfies the required conditions. 


\section{Algebras generated By two inner functions}

If $A$ is a set of positive measure in $\mathbb{T}$, then, by Proposition $\left[2, \overline{\mathbb{C}\left[C \chi_{A}\right]}=\overline{\mathbb{C}[g]}\right.$ for some inner function $g$. This inner function $g$ is unique up to composition with an automorphism of the unit disk. Hence $g$ is unique under the normalization $g(0)=0$. This normalized inner function will be denoted by $g_{A}$.

Lemma 1. Let $g_{1}$ and $g_{2}$ be two inner functions satisfying the following conditions:

(i) There are two disjoint close arcs $J_{1}$ and $J_{2}$ of the unit circle such that $\operatorname{spec}\left(g_{i}\right) \subset J_{i}, i=1,2$;

(ii) $g_{i}$ is univalent on $\mathbb{T} \backslash J_{i}, i=1,2$.

Then for $1 \leq p<\infty$ the algebra $\mathbb{C}\left[g_{1}, g_{2}\right]$ is dense in $H^{p}$.

Proof. We remark that because of the condition (ii) for every subset $A \subset J_{i}$, $g_{k}^{-1}\left(g_{k}(A)\right) \cap\left(\mathbb{T} \backslash J_{i}\right) \subset J_{k}, i, k=1,2, i \neq k$. Let us define the following four sequences of subsets of $\mathbb{T}$. With $D_{0}=\emptyset$, we define inductively

$$
\begin{aligned}
& A_{n}=J_{1} \backslash D_{n-1}, \\
& B_{n}=g_{2}^{-1}\left(g_{2}\left(A_{n}\right)\right), \\
& C_{n}=J_{2} \backslash B_{n}, \\
& D_{n}=g_{1}^{-1}\left(g_{1}\left(C_{n}\right)\right) .
\end{aligned}
$$

It is easy to see that the sequence $B_{n}$ is decreasing, $B_{1} \supset B_{2} \supset \cdots \supset B_{n} \supset \cdots$, and the sequence $D_{n}$ is increasing, $D_{1} \subset D_{2} \subset \cdots \subset D_{n} \subset \cdots$. Also note that for every $n=1,2, \ldots$,

$$
J_{1} \cup J_{2}=B_{n} \cup D_{n}
$$

Let

$$
B=\lim _{n \rightarrow \infty} B_{n}=\bigcap_{n=1}^{\infty} B_{n}, \quad D=\lim _{n \rightarrow \infty} D_{n}=\bigcup_{n=1}^{\infty} D_{n} .
$$

Then (7) implies that

$$
B \cup D=J_{1} \cup J_{2} \quad(\bmod 0) .
$$

Furthermore, $B \cap D=\emptyset$. Indeed, let $z \in D$ and let $k$ be the first index such that $z \in D_{k}$. If $z \in J_{1}$, then $z$ is not in $A_{k+1}$, and, therefore, is not in $B_{k+1}$ which contains $B$. Thus, $z$ is not in $B$. If $z \in J_{2}$, then $z \in C_{k}$ and, hence, is not in $B_{k}$. Since $B_{k}$ contains $B, z$ is not in $B$.

Thus,

$$
\chi_{B}+\chi_{D}=\chi_{B \cup D}=\chi_{J_{1} \cup J_{2}} .
$$

Obviously, $B=g_{2}^{-1}\left(g_{2}(B)\right)$ and $D=g_{1}^{-1}\left(g_{1}(D)\right)$. Therefore, $C\left(\chi_{B}\right) \in \overline{\mathbb{C}\left[g_{2}\right]}$ and $C\left(\chi_{D}\right) \in \overline{\mathbb{C}}\left[g_{1}\right]$. This and (9) yield

$$
\left.C\left(\chi_{J_{1} \cup J_{2}}\right)=C\left(\chi_{B}\right)\right)+C\left(\chi_{D}\right) \in \overline{\mathbb{C}\left[g_{1}\right]}+\overline{\mathbb{C}\left[g_{2}\right]} \subset \overline{\mathbb{C}\left[g_{1}, g_{2}\right]} .
$$

Let $J_{1}^{\prime}$ be an arc such that $J_{1}^{\prime} \supset J_{1}, J_{1}^{\prime} \cap J_{2}=\emptyset$, and $J_{1}^{\prime} \backslash J_{1}$ is an arc. Since $\operatorname{spec}\left(g_{1}\right) \subset$ $J_{1}^{\prime}$ and $g_{1}$ is one-to-one on $\mathbb{T} \backslash J_{1}^{\prime} \subset \mathbb{T} \backslash J_{1}$, (10) implies that $C \chi_{J_{1}^{\prime} \backslash J_{1}} \subset \mathbb{C}\left[g_{1}, g_{2}\right]$. Since $J_{1}^{\prime} \backslash J_{1}$ is an arc, Theorem 2 implies that $\psi \circ C_{J_{1}^{\prime} \backslash J_{2}}$ is an automorphism of $\mathbb{D}$. 
Remark. A pair of functions satisfying the conditions of Lemma 1 can be constructed using Aleksandrov-Clark measures in the following way. Let $\mu_{1}$ and $\mu_{2}$ be two singular measures supported in $J_{1}$ and $J_{2}$, respectively, and $g_{i}, i=1,2$, be the inner function for which $\mu_{i}$ is the Aleksandrov-Clark measure corresponding to $\alpha=1$. Then $g_{1}, g_{2}$ satisfy the conditions of Lemma 1. Conversely, any pair of functions satisfying Lemma 1 comes from such a construction.

Proof of Theorem 3. We note that for any automorphism $\phi$ of the unit disk, the composition operator $C_{\phi}$ is invertible. Therefore, for any pair of functions $\left(g_{1}, g_{2}\right)$ in $H^{\infty}, \mathbb{C}\left[g_{1}, g_{2}\right]$ is dense in $H^{p}$ if and only if $\mathbb{C}\left[g_{1} \circ \phi, g_{2} \circ \phi\right]$ is also dense. Let $g_{i}=\exp \left\{\lambda_{i} \frac{z+w_{i}}{z-w_{i}}\right\}, i=1,2$. If $a$ is the midpoint of the geodesic joining $w_{1}$ and $w_{2}$, then the automorphism of $\mathbb{D}$, which takes $a$ to zero, takes $w_{1}$ and $w_{2}$ into a pair of antipodal points. Without loss of generality we may assume that they are \pm 1 . Furthermore, composition with the automorphism $z \mapsto \frac{a-z}{1-\bar{a} z}$ maps the singular inner function which is determined by the point mass $\lambda$ at $w \in \mathbb{T}$ into the singular inner functions determined by the point mass $\frac{1-|a|^{2}}{|1-\bar{a} w|^{2}} \lambda$ at $\frac{a-w}{1-\bar{a} w}$ (times a unimodular constant). Finally, if $\varphi_{1}=\exp \left\{\rho_{1} \frac{z+1}{z-1}\right\}$ and $\varphi_{2}=\exp \left\{\rho_{2} \frac{z-1}{z+1}\right\}$, then composition with $z \mapsto \frac{z-b}{1-b z}$, where $b=\frac{\sqrt{\rho_{1}}-\sqrt{\rho_{2}}}{\sqrt{\rho_{1}}+\sqrt{\rho_{2}}}$, maps $\varphi_{1}$ and $\varphi_{2}$ into the singular inner function determined by point masses $\sqrt{\rho_{1} \rho_{2}}$ at 1 and -1 , respectively. Thus, we conclude that it suffices to prove Theorem 3 in the case when $w_{1}=1, w_{2}=-1$ and the weights $\lambda_{1}$ and $\lambda_{2}$ are equal. Let us call that common weight $\rho$. We will show that $\mathbb{C}\left[g_{1}, g_{2}\right]$ is dense in $H^{p}$ for $1 \leq p<\infty$ if $\rho<\pi$ and not dense if $\rho>\pi$.

If $\rho>\pi$, then $g_{1}$ and $g_{2}$ do not separate points of $\mathbb{D}$, and therefore $\mathbb{C}\left[g_{1}, g_{2}\right]$ can not be dense in $H^{p}$. Indeed, if $\pi k<\rho \leq \pi(k+1), k \geq 1$, then for

$$
\tau=\frac{\sqrt{\rho^{2}-\pi^{2} k^{2}}-\rho}{\pi k} i
$$

we have $|\tau|<1$ and $g_{1}(\tau)=g_{1}(-\tau), g_{2}(\tau)=g_{2}(-\tau)$.

Now, let $\rho<\pi$. Let $J$ be the closed arc of $\mathbb{T}$ with endpoints $-\frac{\sqrt{\pi^{2}-\rho^{2}}}{\pi} \pm i \frac{\rho}{\pi}$ which contains -1 . It easy to check that $J$ and $-J$ satisfy the conditions of Lemma 1

\section{CONCluding REMARKS}

In closing we would like to discuss two related problems.

Problem 1. Let $\mathcal{A}$ be the subalgebra of $H^{\infty}$ mentioned in Theorem 3, and let the condition of Theorem 3 be satisfied. Is $\mathcal{A}$ weak-* dense in $H^{\infty}$ ?

The motivation for this problem is the question of whether the lattice of $\mathcal{A}$ invariant closed subspaces of $H^{p}$ coincides with the lattice of $z$-invariant subspaces. By Theorem $3 \mathcal{A}$ is dense in every $H^{p}, p<\infty$. But this condition alone is not enough. It was shown to us by the referee that the coincidence of the two lattices is a necessary and sufficient condition for the weak-* density of the algebra in $H^{\infty}$. He also provided an example of an algebra which is dense in every $H^{p}$, but not weak-* dense in $H^{\infty}$.

The case of the algebra $\mathbb{C}\left[e^{\pi \frac{z+1}{z-1}}, e^{\pi \frac{z-1}{z+1}}\right]$ stands alone, because in this case $J_{1}$ and $J_{2}$ are complementary semicircles, and so $\chi_{J_{1} \cup J_{2}}$ is just a constant which is trivially in the algebra, but clearly provides no useful information. 
Problem 2. Is $\mathbb{C}\left[e^{\pi \frac{z+1}{z-1}}, e^{\pi \frac{z-1}{z+1}}\right]$ dense in $H^{p}$ ?

We remark that if $z^{2}$ belongs to the closure of this algebra, then this question has an affirmative answer.

\section{ACKOWLEDGEMENT}

The authors would like to thank the referee for very useful comments. He also showed us that the Problem 1 in section 5 stated as it was in the earlier version of the paper had a negative solution. The present form of this problem was sugested by the referee.

\section{REFERENCES}

[1] A. B. Aleksandrov, Measurable partitions of the circle induced by inner functions, Zap. Nauchn. Sem. Leningrad. Otdel. Mat. Inst. Steklov (LOMI) 149 (1986), Issled. Linein. Teor. Funktsii XV, 103-106, 188; translation in J. Soviet Math. 42 (1988), no. 2, 1610-1613. MR 0849298 (87i:30065)

[2] A. Beurling, On two problems concerning linear transformations in Hilbert space, Acta Math., 81 (1949), 239-255. MR0027954 (10:381e)

[3] R. G. Blumenthal, Holomorphically closed algebras of analytic functions, Math. Scan. 34 (1974), 84-90. MR0380423 (52:1323)

[4] J. B. Garnett, Bounded analytic functions, Academic Press, New York, 1981. MR0628971 (83g:30037)

[5] E. Hewitt, Remark on orthonormal sets in $L^{2}(a, b)$, Amer. Math. Monthly 61 (1954), 249250. MR0060146 (15:631e)

[6] Alexander Izzo, private communication.

[7] M. J. Jaffrey, T. L. Lance, M. I. Stessin, Submodules of the Hardy space over polynomial algebras, Pacific J. Math. 194 (2000), No. 2, 373-392. MR.1760788 (2001e:46097)

[8] J. Jones, Generators of the disk algebra, Ph.D. dissertation, Brown University, 1977.

[9] T. W. Körner, Fourier analysis, Cambridge University Press, Cambridge, 1988. MR0924154 (89f:42001)

[10] W. Seidel, On the distribution of values of bounded analytic functions, Trans. Amer. Math. Soc. 36 (1934), 201-236. MR1501738

[11] N. Sibony, J. Wermer, Generators for $A(\Omega)$, Trans. Amer. Math. Soc. 194 (1974), 103-114. MR0419838(54:7856)

[12] M. I. Stessin, P. J. Thomas, Algebras generated by two bounded holomorphic functions, J. d'Analyse Math. 90 (2003), 89-114. MR2001066 (2004g:46070)

[13] J. Wermer, Rings of analytic functions, Ann. of Math. 67 (1958), 497-516. MR0096817 $(20: 3299)$

[14] J. Wermer, Subalgebras of the disk algebra, Colloque d'Analyse Harmonique et Complexe, Univ. Aix-Marseille I, Marseille, 1977, 7pp. (not consecutively paged). MR0565008 (81e:46035)

Department of Mathematics, Lamar University, Beaumont, Texas 77710

E-mail address: matheson@math.lamar.edu

Department of Mathematics and Statistics, University at Albany, SUny, Albany, New York 12222

E-mail address: stessin@math.albany.edu 